\title{
Psychogeriatric SOS (services-on-screen) - a unique e-health model of psychogeriatric rural and remote outreach
}

Mental health service delivery to rural and remote communities can be significantly impeded by the tyranny of distance. In Australia, rural and remote mental health services are characterized by limited resources stretched across geographically large and socio-economically disadvantaged regions (Inder et al., 2012; Thomas et al., 2012). Internationally, rural and remote area mental health workforce shortages are common, especially in relation to specialist mental health services for older people (McCarthy et al., 2012; Bascu et al., 2012).

In countries that are geographically large and/or under-resourced, the psychogeriatric needs of the rural and remote populations are often addressed by a variety of multidisciplinary clinicians, from primary care, adult mental health, and generalist aged care, or by a small number of psychogeriatric clinicians, sometimes supported by visiting psychiatrists, or some form of teleconferencing (Harte and Bowers, 2011; Mars, 2013). The world literature on e-health solutions specifically focused on older adult mental health is quite sparse (Ramos-Rios et al., 2012). It appears that a significant need remains in geographically large countries and in under-resourced regions for an innovative, efficient and effective e-health model of outreach to enhance existing old age mental health services, whatever may exist.

Efforts to enhance rural and remote mental health service delivery via tele- and video-conferencing, electronic health records, information-based websites, and helplines have demonstrated limited success (Hilty et al., 2004), and although clinically-focused e-health solutions are faster, more economical, and at least as effective as transporting patients and/or clinicians over large distances, the uptake of these services has been surprisingly low (Moffatt and Eley, 2011; Mars, 2013). In Australia, this has led to the proposal that a more powerful solution might be to focus on providing timely support, advice, supervision, training, and education to existing local rural and remote clinicians - to empower them to better manage their patients' psychogeriatric needs at a local level (Access Economics, 2010).

Psychogeriatric SOS (services-on-screen) is a clinician-to-clinician e-health solution that provides multidisciplinary psychogeriatric expertise to clinicians in rural and remote areas of New South Wales (NSW), Australia. The model is based on an integrated, multidisciplinary, team management model of psychogeriatric care currently used in inner city Sydney (St Vincent's Hospital Psychogeriatric Mental Health and Dementia Service), adapted for rural and remote clinicians, and delivered via web-based technology. It provides isolated rural and remote clinicians with the support of a virtual multidisciplinary psychogeriatric team, to assist them to manage their older patients with mental health problems and dementia locally, and as the lead clinicians.

We believe Psychogeriatric SOS has the potential for application well beyond the clinicians of rural and remote NSW, and beyond psychogeriatrics; and through this editorial we are seeking broad consultation, expert opinion and comment regarding the model whilst in its infancy.

\section{The scope of Psychogeriatric SOS}

The St Vincent's Psychogeriatric team comprises two old age psychiatrists, an advanced trainee, a psychiatry registrar, a clinical nurse consultant, two clinical nurse specialists (CNSs), three clinical psychologists, a clinical neuropsychologist, an occupational therapist (OT), a social worker, and four Dementia Service Nurses, totaling 12.5 fulltime equivalents (FTEs). Each member of the team has a high level of general competence in clinical psychogeriatrics, and profession-specific expertise. Our local model of service delivery (see Burke et al., 2008) is based on timely assessment and management of patients in their place of residence (total population over aged 65 years approximately 15,000 ) in collaboration with their general practitioner (GP) and any other health professionals involved, and minimizing the need for emergency department presentation, hospital admission, and respite or permanent placement in a residential aged care facility. We use a team management model of care (see Burke et al., 2013) that focuses on providing multidisciplinary assistance and support as required to the patient, 
their principal clinician(s), other carers and their family.

Psychogeriatric SOS is based on this model, and available to any clinician working in a rural or remote area, in a hospital, community health center, private practice or residential aged care facility. The service commenced as a pilot in January 2015 funded by St Vincent's Hospital, Sydney, the St Vincent's Curran Foundation, and the NSW Institute of Psychiatry/NSW Higher Education and Training Institute. From April, Psychogeriatric SOS received funding from the NSW Ministry of Health Integrated Care Planning and Innovation Fund for 2015/2016. At the time of writing, the service was available to clinicians in three local health districts (LHDs) in rural and remote NSW (total population over aged 65 years approximately 125,000 ) and has been used by GPs, nurses, and psychologists. The Psychogeriatric SOS services include information, discussion, advice, education, supervision, clinical case review, or multidisciplinary case-conferencing, as required.

\section{How Psychogeriatric SOS works}

The initial contact from a rural or remote clinician can be made via phone, fax, email or Internet. They are then asked to register with the service at www.psychogeriatricsos.com.au where they provide background information, and request a web-conference. The request is triaged and a booking made that, as best as possible, matches the requirements of the rural clinician with SOS clinician availability. All clinicians involved in the web-conference are sent an email containing details of the web-conference, including a hyperlink. The rural or remote clinician requires a computer with a web browser, webcam, microphone, speakers, and Internet access to join a web-conference, or can join by phone.

Following the web-conference, a brief report is sent to the clinician(s) who used the service and to the relevant GP(s). All Psychogeriatric SOS occasions of service are reviewed by the St Vincent's Psychogeriatric team at their weekly clinical review meeting to ensure the integrity of the team management model and appropriate clinical governance.

\section{Clinical uses of Psychogeriatric SOS}

Whomever the clinician, whatever the setting, whatever the condition, whatever the phase of management, the rural or remote area clinician is able to request psychogeriatric expertise tailored to particular issues and circumstance. For example
- a community general adult psychologist casemanaging a 72 year old farmer with depression can request advice from a specialist psychogeriatric psychologist regarding cognitive behavior therapy;

- a GP treating a 69 year old woman with dementia who is exhibiting verbally and physically aggressive behavior in a residential aged care facility in a remote town can discuss the use of pharmacological and behavioral management strategies with a clinical nurse specialist and a psychogeriatrician;

- an OT seeing a 63 year old man with lung cancer and brain metastases, who lives in a rural village and wants to disinherit his children, can get advice from a clinical neuropsychologist and a social worker about capacity assessment and guardianship issues; or

- a physiotherapist assessing an 84 year old woman, who lives at home in a small town and is expressing a delusional belief that she is dying of AIDS, can consult a senior psychogeriatric registrar and a psychogeriatric clinical nurse consultant about the options for further assessment and management.

Beyond such specific patient-care scenarios, clinicians in rural and remote areas can access Psychogeriatric SOS for the purpose of regular professional supervision. For example

- a psychologist wishing to run a weekly psychoeducation group for older patients with bipolar disorder can speak to a psychogeriatric clinical psychologist and a clinical nurse specialist for advice on how to structure the sessions, and for weekly supervision following each session; or

- a social worker seeing a patient with advanced Lewy Body dementia, whose family and treating team are in conflict over residential placement, can webconference with a psychogeriatric social worker, clinical neuropsychologist, and OT about possible solutions including guardianship.

\section{Education and training via Psychogeriatric SOS}

Each member of the St Vincent's team has experience in providing lectures, seminars, tutorials, and supervision in the hospital, the community and in residential aged care facilities, in their particular field of psychogeriatric expertise. The aims of our education and training program are to up-skill individual clinicians, and to build capacity within the system in which they work. One of the outcomes is that those clinicians are then able to manage more complex patients before reaching a point where they feel the need to make a referral to our service, and we have found this increases their professional satisfaction, and allows us to be more efficient with our limited resources (Burke et al., 2015).

This model of providing clinical input supplemented by appropriate education and training has been shown to be effective via tele-health 
applications (Hilty et al., 2004; 2006), and is applicable to our goal of up-skilling and empowering rural and remote clinicians through Psychogeriatric SOS. To help achieve this goal a range of resources are available on the website, including book chapters, video-recorded lectures, and copies of or links to recommended assessment and outcome tools. In addition, we have a planned series of web-broadcast "training events" that will be available to small or large groups, live or subsequently as recordings on the website.

\section{The outcomes of Psychogeriatric SOS}

The service will be evaluated using data from pre- and post-service user questionnaires measuring clinical competence and confidence, service usage, patient outcome measures, and measures of costbenefit analysis.

The principal purpose of Psychogeriatric SOS is to provide timely, expert, practical assistance via a team management model to clinicians in rural, remote and under-resourced areas to facilitate the achievement of the best possible mental health outcomes for their patients.

At the individual patient level, the patient's experience of continuity of care should be enhanced, as Psychogeriatric SOS will support the care provided by the local clinician. Patient access to psychogeriatric expertise in rural, remote and under-resourced areas should be improved, and therefore impact on need for travel to seek expert help. This may also lead to a reduced likelihood of admission to a regional facility for assessment and/or respite. Improved assessment, diagnosis or management may impact on illness duration, level of complications, recovery rate, and relapse prevention.

At the population level, the improved access to and provision of psychogeriatric expertise through Psychogeriatric SOS should lead to improved mental health in the older population in rural and remote areas.

At the clinician level, rural and remote clinicians using Psychogeriatric SOS will be up skilled through advice, education, and supervision, creating a greater sense of empowerment, a stronger sense of being supported, and an improved sense of job satisfaction. Their relationships with their patients should be strengthened through achieving better outcomes, and by remaining fully engaged as the lead clinicians. And their professional confidence should be bolstered by enhancement of their knowledge and skills in dealing with a broad range of psychogeriatric issues.
At the LHD level, the provision of previously inaccessible psychogeriatric expertise should enhance local resources in an efficient and cost-effective manner. In the longer term, access to such support could facilitate improved recruitment and retention of local clinicians in rural and remote regions.

\section{The limits and potential of Psychogeriatric SOS}

In 2015, Psychogeriatric SOS is available to rural and remote clinicians in 3 of 17 LHDs in one state of Australia. This limited reach is a function of the current available resourcing for clinician hours on our team. Potentially, the service could be scaled up or down, and provided to one LHD, one or more regions, or one or more states, or an entire country, etc., depending on the level of resourcing. Under the present model (existing team providing a direct service locally plus rural outreach via clinician-to-clinician web-conferencing) the $\mathrm{St}$ Vincent's Hospital Psychogeriatric Mental Health and Dementia Team has increased it's potential reach to people over 65 years by more than $900 \%$ with only a $33 \%$ increase in our multidisciplinary team clinician FTEs. This increase in FTEs has been funded by grants (as above), and during 2015-2017 we aim to develop a business model that will be self-funding and sustainable, and an operational manual to facilitate the translation and implementation of the Psychogeriatric SOS model to other service types.

The service is also currently limited to the area of psychogeriatrics, but we believe the model is "generalizable," and could be used in other sub-specialties in mental health and other branches of medicine, particularly aged-care related services. The Psychogeriatric SOS model focus on clinician-to-clinician activity overcomes the specific challenges and complexities associated with older adults' use of patient-to-clinician telepsychiatry models, specifically sensory loss, and dementia (Ramos-Rios et al., 2012).

Psychogeriatric SOS web-conferencing will work best on up-to-date computer hardware and software, backed up by high-speed broadband Internet. This is a potential limitation in relation to the reach of the service into areas without such information technology (IT) resources. However, at a minimum, Psychogeriatric SOS can be accessed by rural and remote clinicians via a telephone.

Lastly, while the web-conferencing platform used is highly functional, we have encountered issues with government organizations' computer security firewalls blocking the download of enabling software, and with certain users preferring an alternate platform. This has required negotiation 
between the relevant IT departments about compatibility solutions of which a variety exists, but are beyond the scope of this paper.

\section{Conclusions}

In our opinion, the Psychogeriatric SOS model meets a significant need for high quality psychogeriatric services in rural, remote and underresourced areas in NSW by supporting local clinicians to lead the assessment and management of their patients, guided by the expertise of the multidisciplinary clinicians from the St Vincent's Psychogeriatric team. The model could be scaled and translated to suit the needs of other target populations and the service providers, locally, nationally or internationally, and has the potential to significantly increase the reach of the service provider through a relatively small increase in resources. This makes the model potentially applicable to any area, region or country that is geographically large and/or under-resourced.

Again, we would like to invite open dialogue about the potential benefits and limitations of this model in an international context.

We believe Psychogeriatric SOS will make psychogeriatric expertise more readily and inexpensively available to rural and remote populations, and will allow rural patients to be treated in their local environment, by the clinicians who know them best. Therefore, the ultimate beneficiaries of Psychogeriatric SOS will be the patients of the rural and remote clinicians who use the service.

\section{Conflict of interest}

None.

\author{
DAVID BURKe, ${ }^{1}$ AYSE BURKE ${ }^{2}$ AND \\ JACQUELINE HUBER ${ }^{1}$ \\ ${ }^{1}$ Psychogeriatric Service, St Vincent's Hospital, \\ Darlinghurst, New South Wales, Australia \\ Email: David.Burke@svha.org.au \\ ${ }^{2}$ AS Consulting, Bondi, New South Wales, Australia
}

\section{References}

Access Economics. (2010). Financial and externality impacts of high-speed broadband for telehealth. Report for Department of
Broadband, Communications and the Digital Economy. Commonwealth of Australia, Canberra.

Bascu, J., Jeffrey, B., Johnson, S., Martz, D., Novik, N. and Abonyi, S. (2012). Healthy ageing in place: supporting rural seniors' health needs. Online fournal of Rural Nursing and Health Care, 12, 77-87.

Burke, D., Cole, B., Falster, Z., Norrie, L. and Huber, J. (2015). Improving the mental health of residents in aged care facilities through regular consultation, liaison and structured education: a new model of service? Unpublished paper.

Burke, D., Kelly, J., Shahnawaz, Z., Gately, D. and Love, E. (2013). Team management in psychogeriatrics. In: D. Burke and A. Burke (eds.), The St Vincent's Hospital Handbook of Clinical Psychogeriatrics (pp. 3-15). Charlston, SC: CreateSpace Independent Publishing Platform.

Burke, D., Sengoz, A. and Abbott, E. (2008). Integrated mental health care for older people in general practices of inner-city Sydney. In world health organization and world organization of family doctors. Integrating Mental Health into Primary Care: A Global Perspective (pp. 69-76). Geneva: WHO and WONCA.

Harte, J. and Bowers, J. (2011). A Framework for Mental Health Service Delivery in Rural and Remote Queensland: a Literature Review Analysing Models and Treatment Options. Queensland: Centre for Rural and Remote Mental Health.

Hilty, D., Marks, S., Urnes, D., Yellowlees, P. and Nesbitt, T. (2004). Clinical and educational telepsychiatry applications: a review. Canadian fournal of Psychiatry, 49, 12-23.

Hilty, D., Yellowlees, P. and Nesbitt, T. (2006). Evolution of telepsychiatry to rural sites: changes over time in types of referral and in primary care providers' knowledge, skills and satisfaction. General Hospital Psychiatry, 28, 367-373.

Inder, K., Lewin, T. and Kelly, B. (2012). Factors impacting on the well-being of older residents in rural communities. Perspectives in Public Health, 132, 182-191.

Mars, M. (2013). Telemedicine and advances in urban and rural healthcare delivery in Africa. Progress in Cardiovascular Diseases, 56, 326-335.

McCarthy, J., Blow, F., Ignacio, R., Ilgen, M., Austin, K. and Valenstein, M. (2012). Suicide among patients in the Veterans Affairs health system: rural-urban difference in rates, riskd and methods. American fournal of Public Health, 102, S111-S117.

Moffat, J. and Eley, D. (2011). Barriers to the up-take of telemedicine in Australia - a view from providers. Rural and remote Health, 11, 1581 (online at http://www.rrh.org.au).

Ramos-Rios, R., Mateos, R., Lojo, D., Conn, D. and Patterson, T. (2012). Telepsychogeriatrics: a new horizon in the care of mental health problems in the elderly. International Psychogeriatrics, 24, 1708-1724.

Thomas, D., MacDowell, M. and Glasser, M. (2012). Rural mental health workforce needs assessment - a national survey. Rural and Remote Health, 12, 2176 (online at http://www.rrh.org.au). 